\title{
Spectral Response Tuning of Photoconductive Terahertz Emitters With Binary Phase Masks
}

\author{
Christopher M. Collier, Trevor J. Stirling, Shaylene Dekock-Kruger, and Jonathan F. Holzman, \\ Member, IEEE
}

\begin{abstract}
In this work, binary phase masks are designed and implemented for use with a photoconductive terahertz (THz) emitter in order to shape the frequency response of the emitted THz waveforms. Theoretical and experimental results are applied to study the system. The theoretical results are presented in the form of both numerical simulations (to characterize and uniquely identify the underlying time-delayed photogeneration and resulting charge-carrier dynamics) and a simplified model (to conceptualize the system). The theoretical and experimental results are found to agree. It is ultimately shown that the phase delay through the binary phase mask can be used to preferentially allocate power into bands within the THz spectrum. Such results can lay the groundwork for future studies needing precise control of power within the THz spectrum.
\end{abstract}

Index Terms-frequency response, optical beams, photoconductivity, terahertz radiation.

\section{INTRODUCTION}

$\mathrm{O}$ VER the past decades, ultrafast pulsed lasers have enabled numerous advances in pure and applied studiesmany of which rely upon the effective emission and detection of terahertz $(\mathrm{THz})$ radiation [1], [2]. This $\mathrm{THz}$ radiation, spanning $0.1-10 \mathrm{THz}$, has traditionally been difficult to emit and detect [3]-[6], due to the low photon energy, but the advent of sub-picosecond pulse durations with modern ultrafast pulsed lasers has enabled access to the $\mathrm{THz}$ spectrum. We now see $\mathrm{THz}$ radiation applied to communications, given its high carrier frequencies [7]-[9], biomedical imaging, given its low (and typically safer) photon energies [10], [11], and spectroscopy, given its extreme sensitivity to molecular absorption lines [12], [13].

Manuscript received September 11, 2016. This work was supported by the Natural Sciences and Engineering Research Council of Canada (NSERC).

C. M. Collier is with the School of Engineering, University of Guelph, ON, N1G 2W1, Canada (phone: 250-215-5076; e-mail: ccollier@uoguelph.ca).

T. J. Stirling, S. Dekock-Kruger, and J. F. Holzman are with the Integrated Optics Laboratory, The University of British Columbia, Kelowna, BC, V1V 1V7, Canada (e-mail: trevor.stirling@alumni.ubc.ca, shaylene.dekock@alumni.ubc.ca, jonathan.holzman@ubc.ca ).
Given the myriad of $\mathrm{THz}$ applications, the incorporated systems are often required to possess suitable characteristics in terms of (ideally high) emission power [14] and (ideally tunable) spectral response [15]. Photoconductive (PC) THz emitters, i.e., Auston switches [1], [16], have been successful in these regards. In terms of high emission powers, Berry et al. [16] and Jooshesh et al. [17] investigated nanoplasmonic enhancements to improve the optical-to-THz-radiation conversion efficiency in PC THz emitters, while Ropagnol et al. [18] and Mittendorf et al. [19] developed materials and structures for high-power large-area PC THz emitters. In terms of tunable spectral responses, there has also been a great deal of work. Such work has been motivated by applications in communications, for which there is a desire to transmit in lowloss communication bands, such as the 0.8-0.9 THz band [8], [9], and in spectroscopy, for which there is a desire to preferentially excite molecular absorption lines, such as the known 1.36 and $1.82 \mathrm{THz}$ lines for lactose [20]. The THz techniques needed to enable tunable spectral responses have not developed to the extent of the techniques seen for high emission powers, but there is now a greater focus on establishing the underlying techniques needed to control $\mathrm{THz}$ frequency responses.

In response to the need for a tunable spectral response in PC $\mathrm{THz}$ emitters, techniques such as chirped pulse difference frequency generation [15] and temporal delay of optical pulses [21] have been introduced. The temporal delay of optical pulses, in particular, is a very simple but promising approach. The temporal delay has been achieved in $\mathrm{PC} \mathrm{THz}$ emitters by oblique illumination with ultrafast pulsed lasers [22] and by incorporating binary phase masks [21], whereby portions of the cross-sectional area of the illuminating ultrafast pulsed laser beam experience differing optical paths, via differing refractive indices. The $\mathrm{PC} \mathrm{THz}$ emitters with binary phase masks allow for orthogonal illumination by the ultrafast pulsed laser beam, which offers practical advantages in terms of compactness and ease of alignment. However, the design, implementation, and analyses of such tunable $\mathrm{PC} \mathrm{THz}$ emitters have not been explored to a great extent. The proposed work carries out such an exploration.

The proposed work provides an investigation into the 
design, implementation, and analysis of PC THz emitters with binary phase masks being used for spectral response tuning. The binary phase mask covers half of the PC gap-to delay half of the illuminating ultrafast pulsed laser beam-and the thickness of this mask is found to influence the spectral response of the $\mathrm{PC} \mathrm{THz} \mathrm{emitter.} \mathrm{The} \mathrm{far-field} \mathrm{THz}$ emission of the PC THz emitter is analysed via simulations, characterizing the coupled semiconductor and electromagnetic dynamics, and the simulated results are compared to experimental results in the time- and frequency-domains. It is shown that the thickness of the binary phase mask can achieve effective tuning of the spectral response from the $\mathrm{PC} \mathrm{THz} \mathrm{emitter.} \mathrm{It} \mathrm{is} \mathrm{interesting} \mathrm{to}$ note that the proposed technique, which applies a time-domain offset to prescribe the desired frequency-domain response, has a duality to the existing continuous-wave $\mathrm{THz}$ generation technique, which applies a frequency-domain offset to prescribe the desired continuous-wave time-domain response. (Further details on continuous-wave $\mathrm{THz}$ generation are seen in Naftaly et al. [23], which applies two-colour continuouswave photo-mixing for $\mathrm{THz}$ generation, and the references therein.) Ultimately, the proposed technique can support future $\mathrm{THz}$ studies needing power preferentially allocated to specific frequency bands.

\section{ANALYSES}

The overall goal of this work is to design, implement, and analyse PC THz emitters that can enable effective control of their spectral responses. The THz system shown in Figure 1 is implemented to do this. Figure 1(a) shows the full system with the PC THz emitter, while Figure 1(b) shows a close-up of the PC THz emitter. The emitter is comprised of a semi-insulating GaAs substrate, on which there are patterned two electrodes, being $50 / 150 \mathrm{~nm}$ thick $\mathrm{Cr} / \mathrm{Au}$ metal layers. A voltage is applied to the electrodes to establish a bias field across the PC

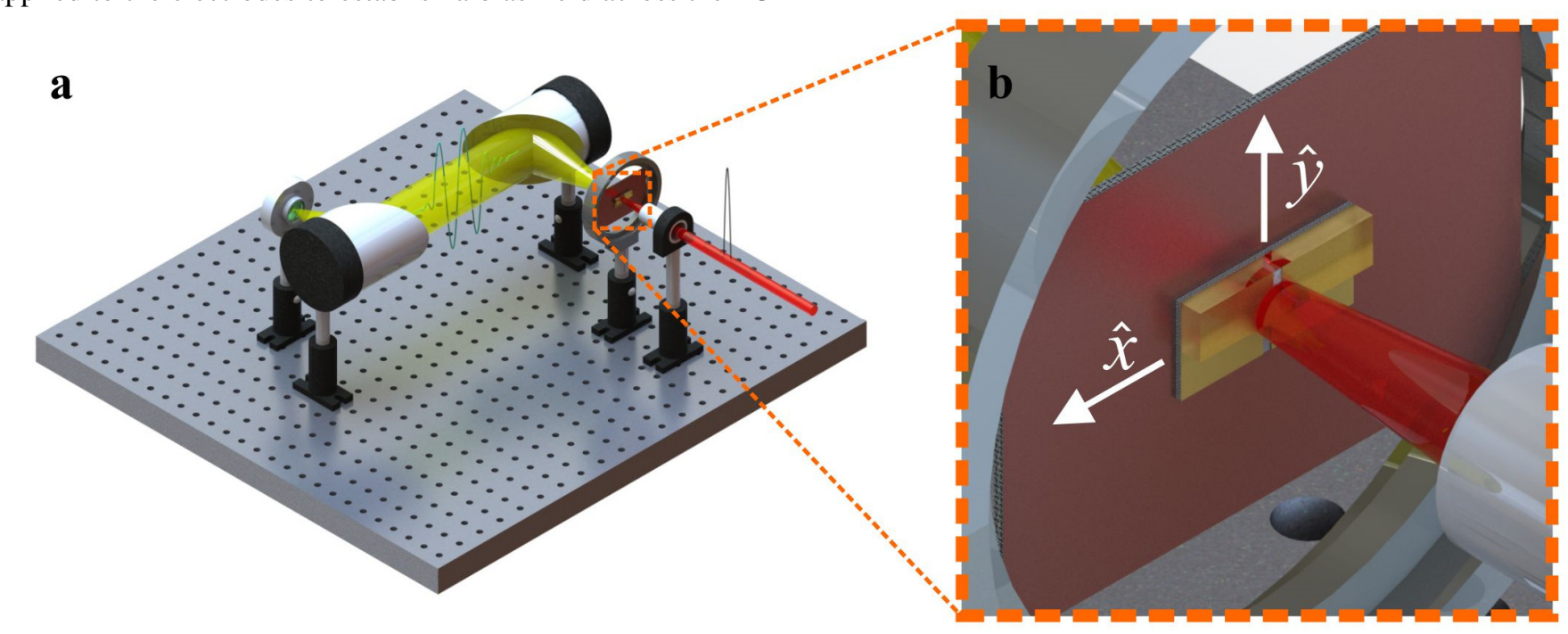

gap, being defined here by the region between the electrodes. The $x$ and $y$ axes shown in Figure 1(b) have an origin at the centre of the PC gap. For such a configuration, the radiated THz radiation will be polarized along the $x$ axis, and it will be emitted preferentially along the $z$ axis, as defined by the crossproduct of the $x$ and $y$ axes in the figure. The $\mathrm{PC} \mathrm{THz} \mathrm{emitter}$ also incorporates a binary phase mask. The binary phase mask is a glass layer with a refractive index of $n$ and a thickness of $d$. It covers the upper (positive y) region of the PC gap, while leaving the lower (negative $y$ ) region of the PC gap uncovered. Upon illuminating the $\mathrm{PC} \mathrm{THz}$ emitter with an ultrashort optical pulse, as shown in the figure, the lower portion of the PC gap is photoexcited before the upper portion of the PC gap. The primary and secondary $\mathrm{THz}$ pulses are separated temporally by the time delay of $\Delta t=(n-1) \cdot d / c$, where $c$ is the speed of light in free space. The PC THz emitter and binary phase mask are shown in Figure 2. Figure 2(a) shows the structure as viewed from above, parallel to the $y$ axis, and Figure 2(b) shows the structure as viewed along the optical axis of the propagating beams, parallel to the $z$ axis. By carefully selecting the time delay of the binary phase mask, the frequency response of the far-field $\mathrm{THz}$ emission is tuned.

To rigorously define the relationship between the applied glass layer and the resulting $\mathrm{THz}$ frequency response, the PC $\mathrm{THz}$ emitter is first analysed via numerical simulations. The simulations describe the charge-carrier photogeneration and transport processes within the PC gap and the electromagnetic radiation that forms from these processes. The work closely follows that of Rodriguez and Taylor [24], [25]. The simulation parameters are defined according to the $\mathrm{PC} \mathrm{THz}$ emitter and binary phase mask in Figure 1 . The PC gap has dimensions in the $x$ and $y$ directions of $L_{\mathrm{x}}=300 \mu \mathrm{m}$ and $L_{\mathrm{y}}=$ $300 \mu \mathrm{m}$, respectively. A bias voltage of $V_{\mathrm{b}}=50 \mathrm{~V}$ forms a bias electric field of $E_{\mathrm{b}} \approx V_{\mathrm{b}} / L_{\mathrm{x}}=1.7 \mathrm{kV} / \mathrm{cm}$ in the PC gap.

Figure 1. A three-dimensional schematic is shown of (a) the complete THz setup with the PC THz emitter having a binary phase mask and (b) a close-up image of the PC THz emitter. The $x$ and $y$ axes for the simulations are shown in (b) with the $z$ axis defined by the cross-product of the $x$ and $y$ axes. The PC THz emitter is comprised of a GaAs PC gap between two metal electrodes and a binary phase mask having a tunable thickness. The mask covers the top half of the illuminated PC gap. The THz emission takes the form of primary and secondary $\mathrm{THz}$ pulses, being separated by the time delay set by the binary phase mask. 


\section{a PC THz emitter viewed from above}

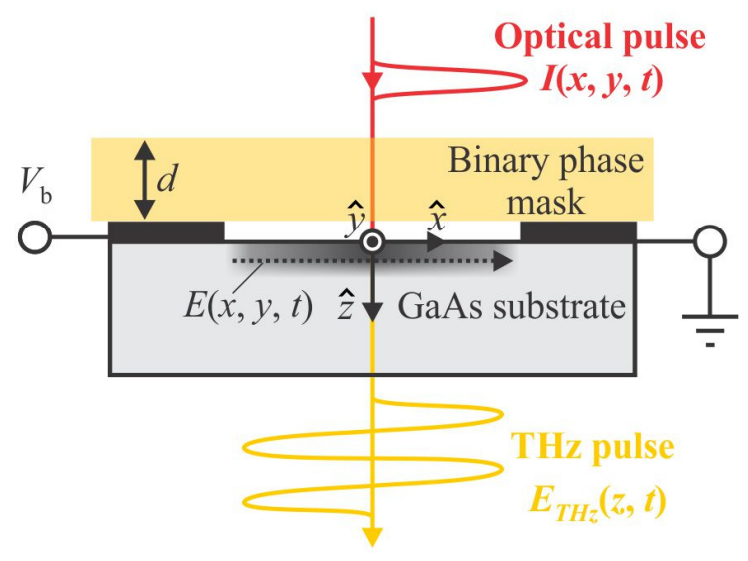

\section{b PC THz emitter viewed along the optical axis}

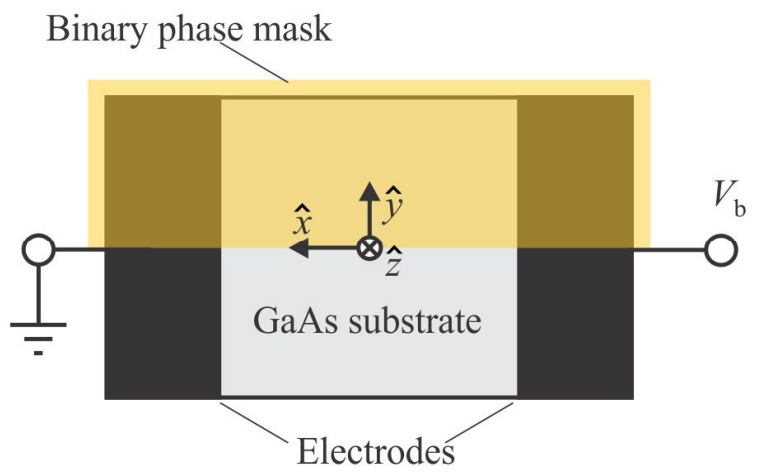

Figure 2. A cross-sectional schematic of the PC THz emitter is shown (a) as viewed from above, being parallel to the $y$ axis, and (b) as viewed along the optical axis of the propagating beams, being parallel to the $z$ axis. The schematic shows the incident optical pulse, with an intensity profile of $I(x, y, t)$, and the resulting THz pulse, with an emitted electric field of $E_{\mathrm{THz}}(z, t)$. The semi-insulating GaAs substrate has two metal electrodes on it, with a bias voltage of $V_{\mathrm{b}}$ establishing the electric field of $E(x, y, t)$ in the PC gap. The binary phase mask has a thickness of $d$.

The PC THz emitter is illuminated by an optical pulse having a Gaussian spatial profile and a Gaussian temporal profile. The optical pulse in the region for $y>0$ (mask) experiences a time delay of $n \cdot d / c$, and the optical pulse in the region for $y<0$ (air) experiences a time delay of $d / c$. This leads to a piece-wise expression for the illuminating intensity on the PC gap, according to

$$
I(x, y, t)=\left\{\begin{array}{l}
\frac{\Phi}{\tau_{p}} \exp \left[-\frac{4 \ln 2 t^{2}}{\tau_{p}^{2}}\right] \exp \left[-\frac{4 \ln 2 x^{2}}{x_{p}^{2}}\right], \quad y<0 \\
\frac{\Phi}{\tau_{p}} \exp \left[-\frac{4 \ln 2(t-\Delta t)^{2}}{\tau_{p}^{2}}\right] \exp \left[-\frac{4 \ln 2 x^{2}}{x_{p}^{2}}\right], y>0
\end{array}\right.
$$

Where $x=y=z=0$ defines the centre of the PC gap. In this expression, $\tau_{\mathrm{p}}=1.3 \mathrm{ps}$ is the temporal full-width-at-halfmaximum (FWHM) of the incident optical pulse and semiconductor response time, $x_{\mathrm{p}}=100 \mu \mathrm{m}$ is the spatial FWHM of the illuminating beam, and $\Phi=5 \mu \mathrm{J} / \mathrm{cm}^{2}$ is the fluence of the illuminating pulse. Note that the illumination intensity is identical in the covered $(y>0)$ and uncovered $(y<$ $0)$ regions, for all but the time delay of $\Delta t=(n-1) \cdot d / c$.

The illuminating intensity is linked to the response of the PC gap by way of semiconductor photogeneration and transport equations. These equations define the surface electron current as

$$
\frac{\partial n_{S}(x, y, t)}{\partial t}=\frac{(1-R)}{h v} I(x, y, t)+\frac{1}{q} \frac{\partial k_{n}(x, y, t)}{\partial x}
$$

and the surface hole current as

$$
\frac{\partial p_{s}(x, y, t)}{\partial t}=\frac{(1-R)}{h v} I(x, y, t)-\frac{1}{q} \frac{\partial k_{p}(x, y, t)}{\partial x}
$$

where $n_{\mathrm{s}}(x, y, t)$ and $p_{\mathrm{s}}(x, y, t)$ are the respective surface electron and hole densities, $k_{\mathrm{n}}(x, y, t)$ and $k_{\mathrm{p}}(x, y, t)$ are the respective surface electron and hole currents, $q$ is the elementary charge, $h$ is Planck's constant, $v=375 \mathrm{THz}$ is the optical frequency of the ultrafast pulsed laser, and $R \approx 0.3$ is the reflectivity of the GaAs surface at the $780 \mathrm{~nm}$ wavelength. For this work, the assumption of $k_{\mathrm{p}}(x, y, t) \approx 0$ is made, as the GaAs hole mobility, $\mu_{\mathrm{p}}=400 \mathrm{~cm}^{2} / \mathrm{V} / \mathrm{s}$, is much less than the GaAs electron mobility, $\mu_{\mathrm{n}}=3000 \mathrm{~cm}^{2} / \mathrm{V} / \mathrm{s}$. This simplifies Equation (3) and allows for an explicit expression for the surface hole density distribution, according to

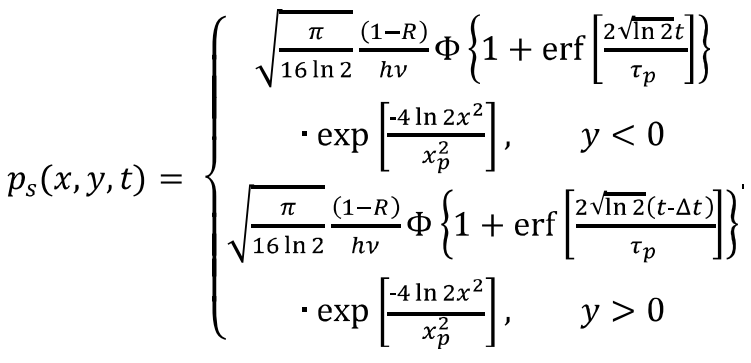

The surface electron current, $k_{\mathrm{n}}(x, y, t)$, can be coupled to the electric field within the PC gap, $E(x, y, t)$, by matching boundary conditions [24]. The result is

$k_{n}(x, y, t)=\frac{\mu_{n} q n_{s}(x, y, t) E(x, y, t)}{\left(1+\sqrt{\varepsilon_{r}}\right)^{-1} \mu_{n} q c \mu_{0} n_{s}(x, y, t)+1}$

where $\varepsilon_{\mathrm{r}}=13$ is the dielectric constant of GaAs, and $\mu_{0}$ is the permeability of free space. An important result is seen here, in that the $\mathrm{THz}$ radiation can be saturated if the illuminating intensity is too high, i.e., the first term in the denominator becomes large. Such saturation is referred to as near-field $\mathrm{THz}$ screening, because it results from the $\mathrm{THz}$ electric field's opposition to the bias electric field.

To create explicit solutions for surface electron density, 
$n_{\mathrm{s}}(x, y, t)$ and electric field within the PC gap, $E(x, y, t)$, Equations (2), (4) and (5) are solved in conjunction with Poisson's Equation,

$\frac{\partial E(x, y, t)}{\partial x}=\frac{q}{\delta \varepsilon_{r} \varepsilon_{0}}\left[p_{s}(x, y, t)-n_{s}(x, y, t)\right]$,

where $\delta=700 \mathrm{~nm}$ is the penetration depth of the illuminating optical beam, and $\varepsilon_{0}$ is the permittivity of free space. This result provides insight into another saturation mechanism for PC THz emitters, being charge-carrier screening. For chargecarrier screening, excessively high charge-carrier densities lead to substantial space-charge fields, from the separating charge-carriers, and these space-charge fields oppose the bias electric field and introduce saturation of the $\mathrm{THz}$ emission.

The solutions for the surface electron and hole densities, surface electron and hole currents, and electric field within the PC gap are solved by way of Equations (1)-(6), and the resulting surface electron current, $k_{\mathrm{n}}(x, y, t)$, is used to define the far-field $\mathrm{THz}$ emission, $E_{\mathrm{THz}}(z, t)$. A Hertzian dipole approximation is used for this. The far-field $\mathrm{THz}$ emission is due to the summation of current contributions across the area of the PC emitter, according to

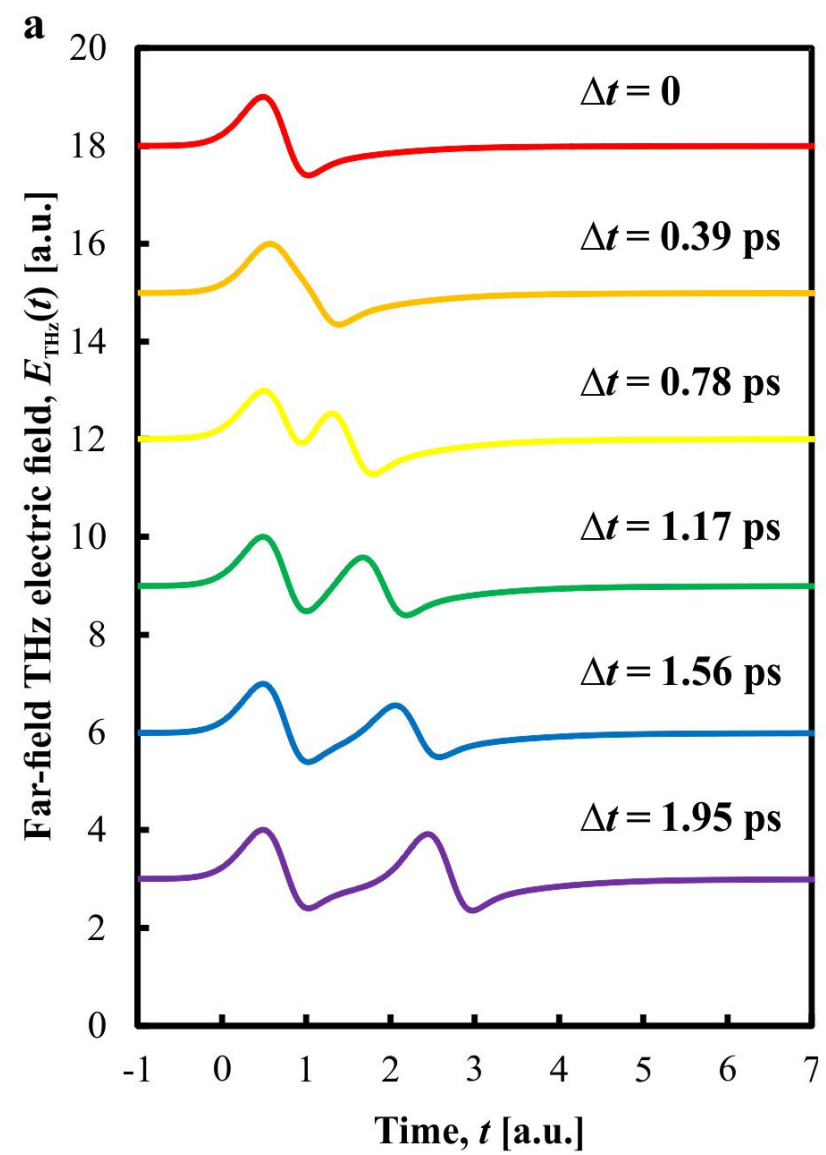

$$
\begin{aligned}
E_{T H z}(z, t)= & \frac{1}{4 \pi \varepsilon_{0} c^{2} z} \frac{d}{d t} \int_{-L_{y} / 2}^{L_{y} / 2} \int_{0}^{L_{x}} k_{n}(x, y, t) d x d y \\
= & \frac{L_{y}}{8 \pi \varepsilon_{0} c^{2} z} \frac{d}{d t} \int_{0}^{L_{x}} k_{n}(x, y>0, t) d x \\
& +\frac{L_{y}}{8 \pi \varepsilon_{0} c^{2} z} \frac{d}{d t} \int_{0}^{L_{x}} k_{n}(x, y<0, t) d x
\end{aligned}
$$

This expression forms the basis for the simulated results of this work.

\section{RESULTS}

The simulation is used to compute time-domain waveforms for the far-field $\mathrm{THz}$ radiation from $\mathrm{PC} \mathrm{THz}$ emitters with a binary phase mask having a refractive index of $n=1.5$ and thicknesses of $d=0,225,450,675,900$, and $1125 \mu \mathrm{m}$. These thicknesses correspond to time delays of $\Delta t=0.39,0.78,1.17$, 1.56 , and $1.95 \mathrm{ps}$, respectively. The results are shown in Figure 3(a). At a thickness of $d=0$, there is no delay due to the binary phase mask, and we see a nominal waveform for the far-field $\mathrm{THz}$ radiation. The unipolar nature of the waveform that one might naïvely expect, given the step-like response of the charge-carrier density, is not present here. A bipolar waveform is formed. The bipolar form is due to the

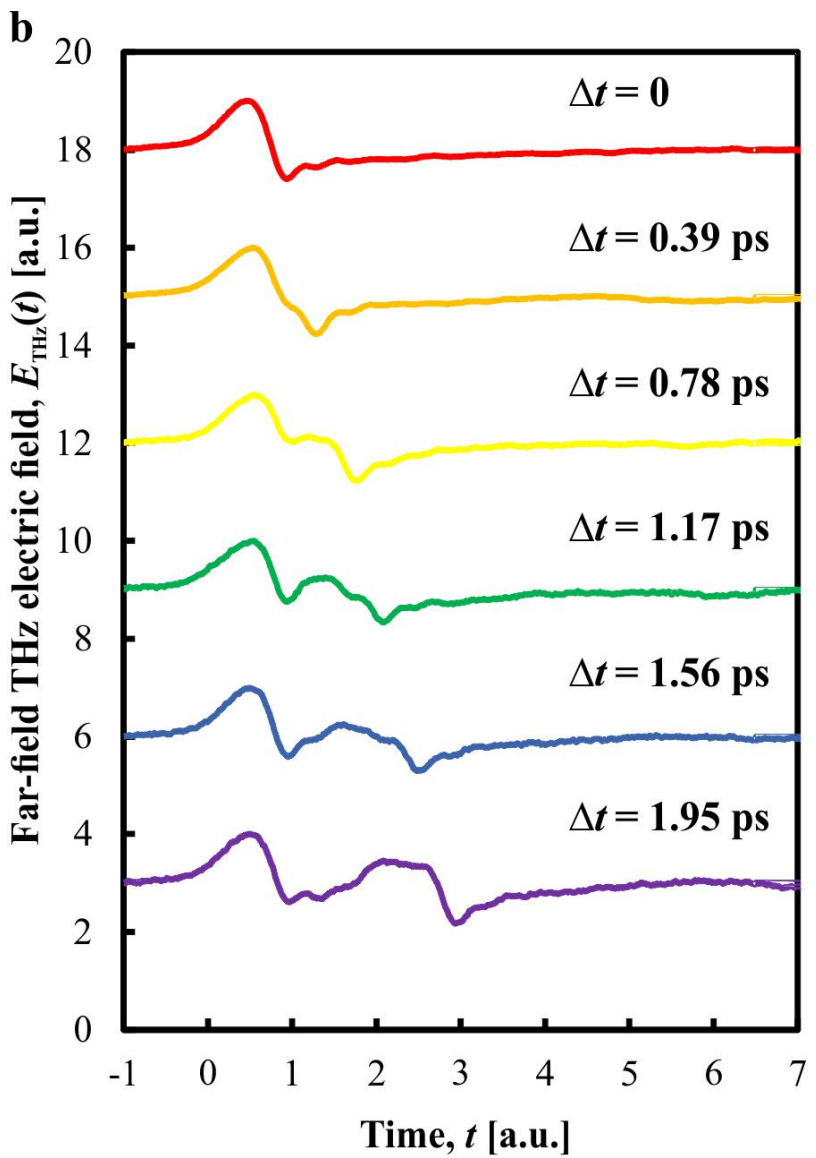

Figure 3. Time-domain results of the far-field $\mathrm{THz}$ electric field, $E_{\mathrm{THz}}(t)$, versus time, $t$, for the PC $\mathrm{THz}$ emitters. The figure shows (a) simulated and (b) experimental results. The PC THz emitters have binary phase masks with time delays of $\Delta t=0.39$, $0.78,1.17,1.56$, and $1.95 \mathrm{ps}$. 
field, due to the separating charge-carriers. (Simulations independently applying near-field $\mathrm{THz}$ screening and chargecarrier screening reveal that the shape of the waveform is defined predominantly by the effects of charge-carrier screening.) As the thickness $d$, and thus the time delay $\Delta t$, is increased, however, varying levels of constructive and destructive interference occur and the shape of the far-field $\mathrm{THz}$ waveform changes. For example, two complete $\mathrm{THz}$ cycles are formed at $\Delta t=1.95 \mathrm{ps}$. It should be noted that the glass-air interfaces of the binary phase mask would introduce internal reflections, in general, but such reflections are negligible (approximately $0.2 \%$ ) for this study. If future implementations of the proposed technique use higher-index materials, however, it may be necessary to consider such reflections and incorporate anti-reflection coatings. To substantiate these theoretical assertions, experiments are carried out with conditions corresponding to those of the above simulations. The experimental results are shown in Figure 3(b). The agreement between the simulated and experimental results is good-in that the experimental waveforms all exhibit the bipolar responses and time delays seen in the simulations.

The frequency-domain responses for the far-field $\mathrm{THz}$ radiation are computed, via Fourier transforms, and are shown in Figure 4. Figures 4(a) and 4(b) show the simulated and

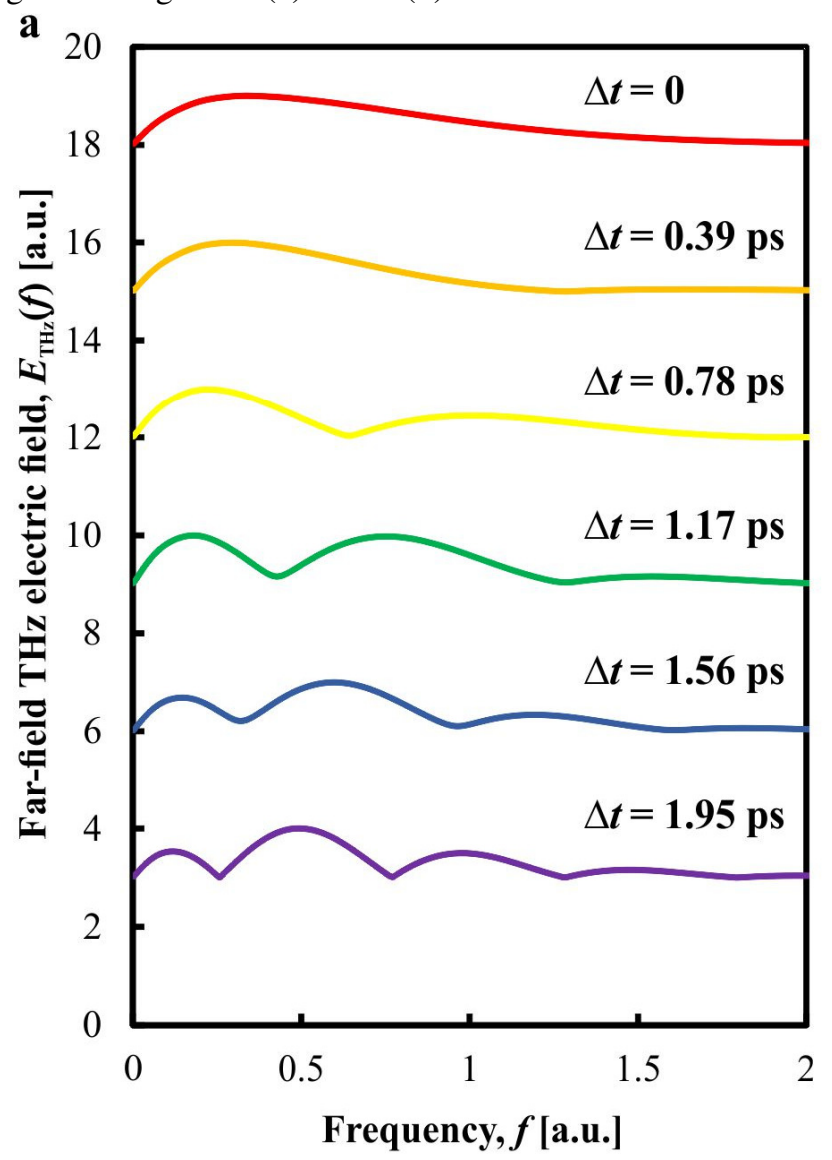

experimental results, respectively. As one would expect, there remains good agreement between the simulated and experimental results in the frequency-domain. Moreover, the effects of spectral response tuning become evident. The application of binary phase masks with increasing time delays form an over-modulation, with peaks and nulls in the spectrum.

The over-modulation in the frequency-domain responses of the far-field $\mathrm{THz}$ radiation can be understood by constructing and analysing a simplified model. We construct such a model by characterizing the frequency-domain responses of Figure 4 by damped sinusoids. The damped sinusoids are each the product of a decaying exponential (for which the Fourier transform is a Lorentzian pulse) and a sinusoid with a periodicity in the frequency-domain of $\Delta f=2 / \Delta t$ (for which the Fourier transform is two delta functions separated by $\Delta t$ ). The corresponding time-domain waveforms of the far-field $\mathrm{THz}$ radiation are then found, via convolution, to be two Lorentzian pulses separated by $\Delta t$. Ultimately, this simplified model suggests that two pulses in the time-domain, separated a delay of $\Delta t$, yield periodicity in the frequency-domain of $\Delta f=2 / \Delta t$. Thus, the simulated and experimental results, having two pulses in the time-domain separated by a delay of $\Delta t$, exhibit periodicity (i.e., peaks and nulls) in the frequency-domain.

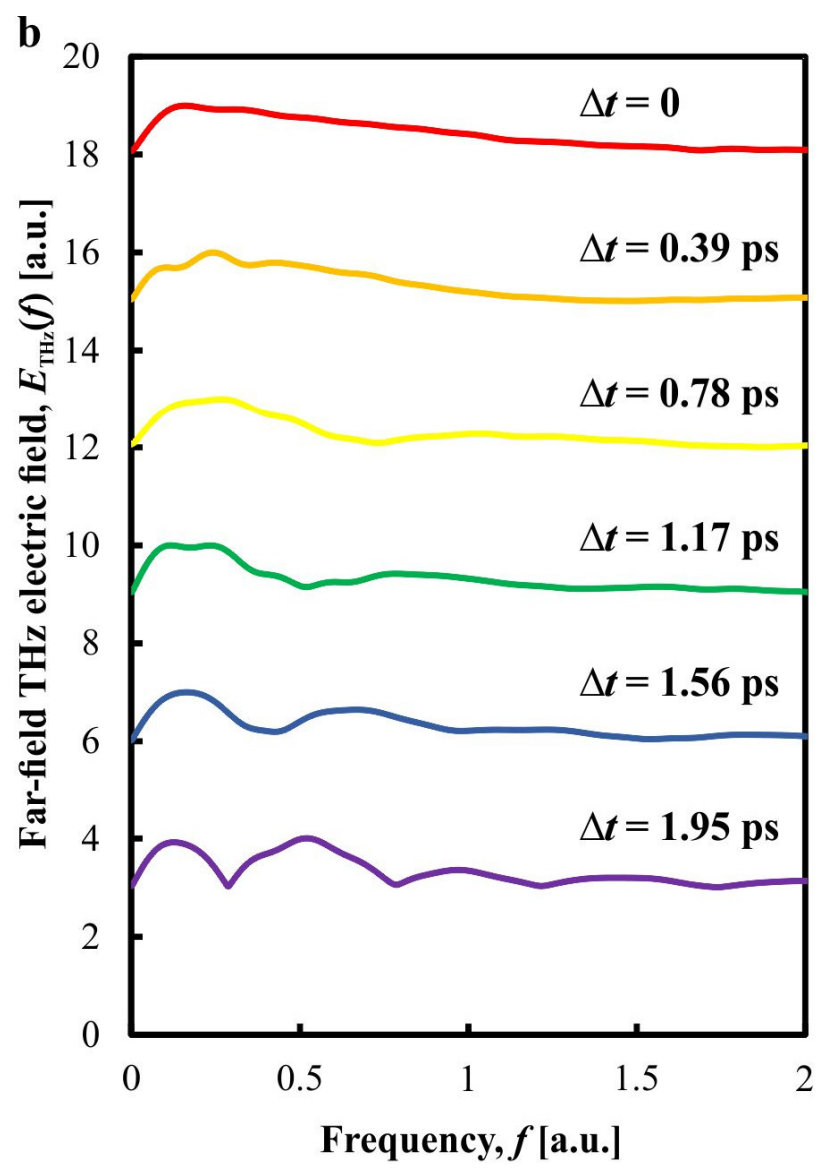

Figure 4. Frequency results of the far-field THz electric field, $E_{\mathrm{THz}}(f)$, versus frequency, $f$, for the $\mathrm{PC} \mathrm{THz}$ emitters. The figure shows (a) simulated and (b) experimental results. The PC THz emitters have binary phase masks with time delays of $\Delta t=0.39$, $0.78,1.17,1.56$, and $1.95 \mathrm{ps}$. 
The understanding brought about through the simplified model can be linked to the simulated and experimental results. Figure 5 does this by showing discrete data points for the delay times predicted by the simulated and experimental results, as a function of the true time delays, $\Delta t$. The figure also shows the simple linear trendline that is predicted by the simplified model. The frequency-domain responses for the simulated and experimental results are curve fit to the damped sinusoid of the model, and the optimal curve fits are used to generate $\Delta t_{\text {fit }}$ values for each of the six simulated/experimental conditions, i.e., the six $d$ and $\Delta t$ values. The fit values for the simulated and experimental time delays are plotted in the figure as a function of the true time delay and are seen to be in good agreement, i.e., $\Delta t_{\text {fit }} \approx \Delta t$. Moreover, the simulated and experimental results are in close proximity to the linear trendline that would be expected via the simplified model. The simulated and experimental results agree with the results of the simplified model, with R-squared values of $R^{2}=0.995$ and 0.996 , respectively.

Ultimately, this simplified model lets us see the underlying effects of the binary phase mask. The mask creates a phase shift in half the $\mathrm{THz}$ emission, with respect to the other half, with the two portions of the $\mathrm{THz}$ emission exhibiting beating in the far-field. The extent to which the phase shift is applied, i.e., the value of the time delay, can be used to form an overmodulation in the frequency response-and thus can be used to preferentially allocate power in $\mathrm{THz}$ bands.

\section{CONCLUSION}

In this work, binary phase masks were designed and implemented for use with a PC $\mathrm{THz}$ emitter. The system was analysed by way of simulations and experiments. The

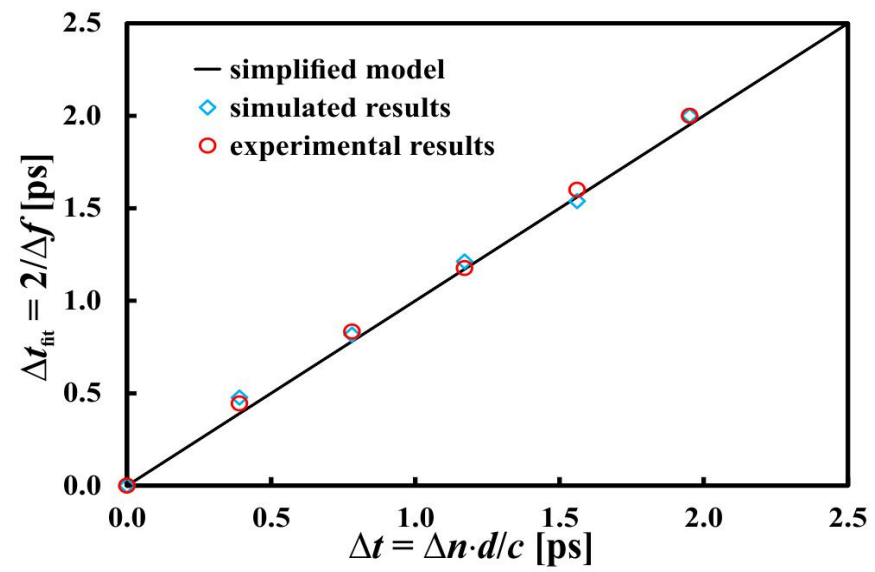

Figure 5. Time delay for fitting with the model, $\Delta t_{\text {fit }}=2 / \Delta f$, versus the true time delay, $\Delta t=\Delta n \cdot d / c$. The figure shows results of the simplified model (black solid line), simulated results (blue diamonds), and experimental results (red circles) for $\mathrm{PC} \mathrm{THz}$ emitters with varying thicknesses for the binary phase masks. The simulated and experimental results for each time delay, $\Delta t$, are fit with damped sinusoids to define $\Delta t_{\text {fit }}$. The linear trendline of $\Delta t_{\mathrm{fit}}=\Delta t$ depicts the predictions of the simplified model. simulated and experimental results were in strong agreement, and were interpreted in terms of a simplified model. It was shown that the binary phase mask can preferentially tune the phase response of the emitted $\mathrm{THz}$ radiation-and thus preferentially allocate power into bands within the $\mathrm{THz}$ spectrum. These findings can be applied in future $\mathrm{THz}$ systems needing precise control of power within the $\mathrm{THz}$ spectrum.

\section{REFERENCES}

[1]D. H. Auston, K. P. Cheung, and P. R. Smith, "Picosecond photoconducting Hertzian dipoles," Appl. Phys. Lett., vol. 45, pp. 284286, Aug. 1984.

[2]C. M. Collier, M. H. Bergen, T. J. Stirling, M. A. DeWachter, and J. F. Holzman, "Optimization processes for pulsed terahertz systems," Appl. Opt., vol. 54, pp. 535-545, Jan. 2015.

[3]R. Chakkittakandy, J. A. W. M. Corver, and P. C. M. Planken, "Quasinear field terahertz generation and detection," Opt. Express, vol. 16, pp. 12794-12805, Aug. 2008.

[4]H. Pahlevaninezhad, B. Heshmat, and T. E. Darcie, "Advances in terahertz waveguides and sources," IEEE Photon., J. vol. 3, pp. 307-310, Apr. 2011.

[5]D. Mittleman, "Spectroscopic techniques and results", in Sensing with Terahertz Radiation, Heidelberg, Germany: Springer-Verlag, 2003, pp. $51-75$.

[6]M. C. Nuss and J. Orenstein, "Terahertz time-domain spectroscopy", in Millimeter and Submillimeter Wave Spectroscopy of Solids, Heidelberg, Germany: Springer-Verlag, 1998, pp. 7-50.

[7]R. Piesiewicz, M. Jacob, M. Koch, J. Schoebel, and T. Kurner, "Performance analysis of future multigigabit wireless communication systems at $\mathrm{THz}$ frequencies with highly directive antennas in realistic indoor environments," IEEE J. Sel. Top. Quantum Electron., vol. 14, pp. 421-430, Apr. 2008.

[8]R. Piesiewicz, T. Kleine-Ostmann, N. Krumbholz, D. Mittleman, M. Koch, J. Schoebel, and T. Kurner "Short-range ultra-broadband terahertz communications: Concepts and perspectives," IEEE Antennas Propogat. Mag., vol. 49, pp. 24-39, Dec. 2007.

[9] T. Kleine-Ostmann, and T. Nagatsuma, "A review on terahertz communications research," J. Infrared Milli. Terahz. Waves, vol. 32, pp. 143-171, Feb. 2011.

[10] E. Pickwell, B. E. Cole, A. J. Fitzgerald, M. Pepper, and V. P. Wallace, "In vivo study of human skin using pulsed terahertz radiation," Phys. Med. Biol., vol. 49, pp. 1595-1607, Apr. 2004.

[11] S. Y. Huang, Y. X. J. Wang, D. K. W. Yeung, A. T. Ahuja, Y-T. Zhang, and E. Pickwell-MacPherson, "Tissue characterization using terahertz pulsed imaging in reflection geometry," Phys. Med. Biol., vol. 54, pp. 149-160, Dec. 2008.

[12] C. Yu, S. Fan, Y. Sun, and E. Pickwell-MacPherson, "The potential of terahertz imaging for cancer diagnosis: A review of investigations to date," Quantitative Imaging in Medicine and Surgery, vol. 2, pp. 33-45, Mar. 2012.

[13] Y. C. Shen and P. F. Taday, "Development and application of terahertz pulsed imaging for nondestructive inspection of pharmaceutical tablet," IEEE J. Sel. Top. Quantum Electron., vol. 14, pp. 407-415, Apr. 2008.

[14] S. Winnerl, "Scalable Microstructured Photoconductive Terahertz Emitters," J. Infrared Milli. Terahz. Waves, vol. 54, pp. 149-160, Apr. 2012.

[15] J. Krause, M. Wagner, S. Winnerl, M. Helm, and D. Stehr, "Tunable narrowband $\mathrm{THz}$ pulse generation in scalable large area photoconductive antennas," Opt. Express, vol. 19, pp. 19114-19121, Sept. 2011.

[16] C. W. Berry, N. Wang, N. R. Hashemi, N. Unlu, and M. Jarrahi, "Significant performance enhancement in photoconductive terahertz optoelectronics by incorporating plasmonic contact electrodes," Nat. Commun., vol. 4, pp. 1622, Mar. 2013.

[17] A. Jooshesh, V. Bahrami-Yekta, J. Zhang, T. Tiedje, T. Darcle, and R. Gordon, "Plasmon-enhanced below bandgap photoconductive terahertz generation and detection," Nano Lett., vol. 15, pp. 8306-8310, Nov. 2015.

[18] X. Ropagnol, F. Blanchard, T. Ozaki, and M. Reid, "Intense terahertz generation at low frequencies using an interdigitated ZnSe large aperture 
photoconductive antenna," Appl. Phys. Lett., vol. 103, pp. 161108, Oct. 2013.

[19] M. Mittendorf, M. Xu, R. J. Dietz, H. Künzel, B. Sartorius, H. Schneider, M. Helm, and S. Winnerl, "Large area photoconductive terahertz emitter for $1.55 \mu \mathrm{m}$ excitation based on an InGaAs heterostructure," Nano Lett., vol. 24, pp. 214007, Apr. 2013.

[20] Y. Zhang, X. Zhang, S. Li, J. Gu, Y. Li, Z. Tian, C. Ouyang, M. He, J. Han, and W. Zhang, "A broadband THz-TDS system based on DSTMS emitter and LTG InGaAs/InAlAs photoconductive antenna detector," Sci. Rep., vol. 6, pp. 26949, May 2016.

[21] X. Ropagnol, R. Morandotti, T. Ozaki, and M. Reid, "THz pulse shaping and improved optical-to- $\mathrm{THz}$ conversion efficiency using a binary phase mask," Opt. Lett., vol. 36, pp. 2662-2664, July, 2011.

[22] M. Jarrahi, "Terahertz radiation-band engineering through spatial beamshaping," IEEE Photonics Technol. Lett., vol. 21, pp. 830-832, June 2009.

[23] M. Naftaly, M. R. Stone, A. Malcoci, R. E. Miles, and I. Camara Mayorga, "Generation of CW terahertz radiation using two-colour laser with Fabry-Perot etalon," Electron. Lett., vol. 41, pp. 128-129, Mar. 2005.

[24] G. Rodriguez and A. J. Taylor, "Screening of the bias field in terahertz generation from photoconductors," Opt. Lett., vol. 21, pp. 1046-1048, July 1996.

[25] A. J. Taylor, P. K. Benicewicz, and S. M. Young, "Modeling of femtosecond electromagnetic pulses from large-aperture photoconductors," Opt. Lett., vol. 18, pp. 1340-1342, Aug. 1993.

Christopher M. Collier received the B.A.Sc. and Ph.D. degrees in electrical engineering from the University of British Columbia, Kelowna, BC, Canada, in 2011 and 2016, respectively. He is currently an Assistant Professor with the School of Engineering, University of Guelph, Guelph, ON, Canada.

Trevor J. Stirling is pursuing the B.A.Sc. degree in electrical engineering from the University of British Columbia, Kelowna, BC, Canada.

Shaylene Dekock-Kruger is pursuing the B.A.Sc. degree in electrical engineering from the University of British Columbia, Kelowna, BC, Canada.

Jonathan F. Holzman (M'07) received the B.Sc. degree in engineering physics and the Ph.D. degree in electrical engineering from the University of Alberta, Edmonton, AB, Canada, in 1998 and 2003, respectively. He was a Post-Doctoral Research Fellow with the Department of Information Technology and Electrical Engineering, Swiss Federal Institute of Technology, Zürich, Switzerland, from 2004 to 2005. He is currently an Associate Professor with the School of Engineering, University of British Columbia, Kelowna, BC, Canada. 